\title{
Energy Efficient External Sensing in Multichannel Cooperative Cognitive Radio
}

\author{
Hemlata Patil $^{1, *}$, A. J. Patil ${ }^{2}$ and S. G. Bhirud ${ }^{3}$ \\ ${ }^{1}$ Ramrao Adik Institute of Technology, Mumbai University, India \\ ${ }^{2} S G D C O E$, North Maharashtra University, India \\ ${ }^{3}$ VJTI, Mumbai, India \\ *Corresponding Author: hema.patil08@gmail.com
}

Received 30 June 2016; Accepted 19 September 2016;

Publication 10 October 2016

\begin{abstract}
In this paper energy-efficient spectrum sensing for multichannel cooperative cognitive radio is proposed. The most essential characteristic of Cognitive Radio is spectrum sensing. The energy consumption of spectrum sensing is mainly affected by how often the primary channel sensed by secondary users. By keeping this point in mind, proposed approach tried to reduce sensing time and also improves the detection precision by quality energy detection. In conventional spectrum sensing secondary users sense the spectrum without disturbing primary user and try to find a vacant slot individually or cooperatively. In doing this a lot of energy is wasted in sensing by secondary users. Cooperation also increases the amount of energy spent in sensing the spectrum as well as in reporting the message about the spectrum vacancy to fusion center. In the proposed method the spectrum sensing is done by external node. It is verified that if the external node is used for channel sensing the tradeoff between sensing/transmission and wait/switch is enhanced. This proposed method reduces energy consumption and improves throughput. Analytical expressions for above method are derived. This work suggests an effective energy saving scheme compared to conventional schemes.
\end{abstract}

Journal of Green Engineering, Vol. 6, 1-12.

doi: 10.13052/jge1904-4720.622

(c) 2016 River Publishers. All rights reserved. 
Keywords: Cooperative Cognitive Radio, Energy Efficient, External Sensing, Multichannel.

\section{Literature Survey}

As the cellular communication has evolved from $1 \mathrm{G}$ to $5 \mathrm{G}$ the spectrum demand has risen up enormously. FCC says that almost $50 \%$ bands are vacant and can be used dynamically to suffice today's need of spectrum scarcity [1]. The motive behind this is to use the available spectrum wisely without disturbing the operation of primary user (PU). Cognitive Radio is a model which uses primary user's radio spectrum efficiently by sharing with secondary users dynamically [2]. Spectrum sensing is an important task in cognitive radio. Multipath fading, noise uncertainty, and shadowing is present in spectrum sensing. To combat these issues cooperative sensing is used [3]. In conventional cooperative sensing all secondary users will try to sense spectrum individually and combine their results in fusion detector. In hard combined rule only one bit is used for decision making which sometimes weakens the results, this is overcome by using soft combination technique in [4] which still gives the overheads. According to the findings of [5] secondary user cannot sense and access the channel at same time consequently secondary user can only transmit data or wait on current channel without transmission in the same frame. This problem is addressed where external node [6] is allowed to sense the channel in its sensing time and reports its result to secondary users. In turn secondary users transmit their data in their transmission time thus effectively saving the sensing time and improving the energy efficiency. Previous work demonstrated as the sensing time increases the sensing accuracy is improved [7] in single channel. On the other hand, SUs which are mostly mobile devices should be energy-efficient they use their battery power. Therefore, from the energy efficiency perspective, the more time is spent on sensing the more energy is consumed for overhead and less time remains for transmission. The throughput of the network is a function of the detection accuracy. Hence, there is a trade-off between sensing and transmission durations for both throughput and energy efficiency.

\section{System Model}

Consider a cognitive radio network of ' $\mathrm{K}$ ' number of secondary users and ' $M$ ' primary user channel. The External network (EN) consists of single node placed in vicinity of primary user and employs narrow band periodic sensing 
in round robin fashion. The main goal is to sense all $\mathrm{M}$ channels with minimum energy and sufficient accuracy.

In conventional spectrum sensing CR sense the channel and identify the vacant channel and use them for transmission of data. But in this technique external node senses the spectrum of PU and broadcast the message to other secondary users about the availability of spectrum, suppose it finds the first slot to be empty, it sends message to all SU's about the vacancy of empty spectrum slot. It is assumed that this message reaches to all SU's. The secondary users will be given chance sequentially according to their priority to use the channel. Suppose we consider according to their priorities they have been assigned as SU1, SU2 and SU3. SU's are allowed to use spectrum sequentially so SU1 will start its data transmission with first available slot following that SU2 and then SU3. The delay in this case is caused by only waiting period of external node otherwise the secondary users are busy transmitting their data in the given available slots. As primary user comes in, the secondary user has to stop its transmission and switch to next current available channel. The decision given by external node is assumed to free of error. The secondary users are considered in wake/sleep mode depending upon whether they have to transmit data or wait for the message from the external node. Each frame of external node consists of a sensing slot and a reporting/transmission slot. Primary user is assumed to be in on-off traffic model [6],

Hypothesis model for energy detection can be given as [6];

$$
\begin{aligned}
& x_{r}(t)=n_{c}(t), \quad 0<t<T \quad \text { under } \mathrm{H}_{0} \\
& x_{r}(t)=h_{c} s_{p}(t)+n_{c}(t), \quad 0<t<T \quad \text { under } \mathrm{H}_{1}
\end{aligned}
$$

Where, $x_{r}(t)$ - signal received at the external node, $n_{c}(t)$ is zero mean additive white Gaussian noise (AWGN), $h_{c}$ is the channel gain of the sensing channel and $s_{p}(t)$ is transmitted primary user signal. The external network uses two hypotheses

$\mathrm{H}_{0}$ : Spectrum is idle

$\mathrm{H}_{1}$ : Spectrum is occupied

The energy detection method is used to sense the channel, the average received signal to noise ratio SNR of the PU signal is $\gamma$. The false alarm probability and detection probability under non fading environment are given by [8].

$$
\begin{aligned}
& P f n=\frac{\Gamma\left(u, \frac{\lambda}{2}\right)}{\Gamma(u)} \\
& P d n=Q u(\sqrt{2 \gamma, \sqrt{\lambda}})
\end{aligned}
$$


Where,

$P f n$ and $P d n$ are the false alarm probability and detection probability of the external node in the design.

$u$ is time bandwidth product.

$\Gamma(a, x)$ is incomplete gamma function given by $\Gamma(a, x)=\int_{x}^{\infty} t^{a-1} e^{-t} d t$, $\lambda$ is the threshold of the energy detector

$Q u$ is generalized Marcum $\mathrm{Q}$ function given by

$$
Q u(a, x)=\frac{1}{a^{u-1}} \int_{x}^{\infty} t^{u} e \frac{t^{2}+a^{2}}{2} I u-1(a t) d t
$$

The primary user's channel is accessed by external node. Let $\beta$ be the probability that the channel is occupied and $1-\beta$ is the probability that the channel is vacant. The different circumstances under channel sensing are [9]; the probability of the channel that it is used by primary user is $\beta \cdot P d n$. The probability of the channel that it is used by primary and secondary user is $\beta \cdot(1-P d n)$. The probability of the channel that it is used by secondary users is $(1-\beta) \cdot(1-P f n)$. The probability of the channel that neither one is using $(1-\beta) \cdot P f n$. Thus, the average channel throughput $C n$ is given by

Thus, the average channel throughput is given by Cavg

$$
\begin{aligned}
\text { Cavg }= & \beta P d n \cdot(C p+C n)+\beta(1-P d n) \cdot(C p s+C s p+C n) \\
& +(1-\beta) \cdot(1-P f n) \cdot(C s+C n)+(1-\beta) P f n \cdot C n
\end{aligned}
$$

Where $C p, C s, C n$ is the throughput of primary user, secondary user and external node respectively. $C p s$ is the throughput of $\mathrm{PU}$ when $\mathrm{SU}$ is present and $C s p$ is the throughput of SU when PU is present. The total throughput for the system will be

$$
C_{\text {tot }}=\sum_{t=1}^{M} \operatorname{Cavg}^{t} \quad \text { where } t=1,2 . . M
$$

In proposed method external node senses the spectrum alone so less sensing time is needed compared to conventional cooperative spectrum sensing. As secondary users are not sensing they get more time for transmission. On the other hand as sensing information is received from EN the wait time of secondary users is reduced in turn decreasing delay which helps to increase the throughput. 
The average energy consumption by the network is considered. The different scenarios are

- The probability PU is correctly detected as busy is $\beta \cdot P d n$. The energy spend in this situation is Ens $+E n r+E p$

- The probability that PU is falsely detected as vacant is $(1-\beta) \cdot(1-P d n)$ and in this case the energy spend the energy spend in this situation is $E n s+E n r+E p s+E s p$

- The probability that PU was falsely detected as busy is $\beta \cdot P f n$ and the energy spend in this situation is Ens + Enr

- The probability that PU is correctly detected as vacant is $(1-\beta) \cdot(1-$ $P(n)$ and the energy spend in this situation is Ens $+E n r+E s$

Where Ens, Enr is the energy required by the external node for sensing the PU and reporting the information to SU's respectively. Ep is the energy required by the PU for data transmission. Es is the energy required by the $\mathrm{SU}$ for data transmission. $E n$ is the energy required by external node i.e., $E n=E n s+E n r$

$$
\begin{aligned}
\text { Eavg }= & \beta P d n \cdot(\text { Ens }+ \text { Enr }+E p)+\beta(1-P d n) \cdot(\text { Ens }+E n r+E p+E s) \\
& +(1-\beta)(1-P f n) \cdot(E n s+E n r+E s) \\
& +(1-\beta) P f n \cdot E n \\
E_{t o t}= & \sum_{t=1}^{M} E^{M} g^{t}
\end{aligned}
$$

The states related to channel sensing are busy state, idle state and switching state. These probabilities are explained in detail in the following session.

a. Current channel is idle

If the current channel is idle, SU will transmit its data in a given slot. The probability of idle channel Pidle is given as

$$
\text { Pidle }=(1-\beta)(1-P f n)+\beta(1-P d n)
$$

b. All other channels are busy

If all $\mathrm{M}$ channels are busy, SU will wait on the current channel until predefined sensing time $\tau s n$ is over. Switching channels consumes more energy than just waiting and the delay problem is not solved.

The probability of busy state Pbusy is given as

$$
\text { Pbusy }=((1-\beta) P f n+\beta P d n)^{\wedge}(M-1)
$$


c. At least one channel is idle

In this situation, the SU has to decide to switch to the idle channel or keep waiting in current channel. If the SU switches to the idle channel, the throughput increases but consumes more energy. If the SU stays in current channel and power off during $\mathrm{T}$ duration, the energy consumption is lower. However, delay is increased and throughput reduces.

EN senses the channel as it finds the current channel to be busy it moves to next channel to find idle one. It keeps moving in such fashion until it finds one. EN needs to decide when to switch or wait on current channel. In such a case, we assume that the EN waits on the current channel with a probability of $P s$, or switches to another vacant channel with a probability of $1-P s$.

At the same time when EN is waiting on current channel secondary users also wait on current channel and stop its transmission.

$$
\begin{aligned}
P s w & =(1-\text { Pidle })(1-\text { Pbusy })(1-P s) \\
P t & =\text { Pidle }+ \text { Psw. }
\end{aligned}
$$

Where $P s w$ and $P t$ are switching probability and data transmisision probability of SU.

The Delay in this case can occur because of sensing time of external node and waiting time of external node and secondary users.

$$
D=N^{*} \tau s n^{*}+N^{*} T^{*} P w t+(k-1) T^{*} P w t
$$

Where, Pwt is the total probability that the EN waits on the current channel. For each frame, EN has to wait for $\tau s n$, so first part of the formula describes the delay for sensing in $\mathrm{N}$ frames. If $\mathrm{EN}$ waits on the current channel with the probability of $P w t$, the delay in $\mathrm{N}$ frames is NTPwt. Similarly SU has to wait on current channel until it gets vacant position so its delay will be $(K-1) N T P w t$.

$$
\text { Pwt }=(1-\text { Pidle }) \text { Pbusy }+(1-\text { Pidle })(1-\text { Pbusy }) \text { Pstop }
$$

Similarly, the energy consumption under multi-channel can be calculated by using suitable detection probabilities. Throughput and energy consumption are modified including switching probabilities and can be written as

$$
\begin{aligned}
& C=C_{a v g}+P s w(T s+T n) \\
& E=E_{a v g}+P s w E s w+P t E t x
\end{aligned}
$$


Thus with different detection probabilities and switching probabilities the external sensing in multichannel cognitive radio performs better than conventional cooperative sensing.

\section{Simulation Result}

The primary user is considered to have BPSK modulated wave with bandwidth of $2 \mathrm{MHz}$. The values of parameters are taken from [5, 9] for fair comparison. The sampling frequency $f_{s}$ is same as bandwidth and sensing duration to be $5 \mathrm{~ms}$, therefore the time bandwidth product $\mathrm{u}$ comes out to be 10 . The value of $\beta$ i.e., the probability of channel being occupied is taken as 0.5 . As the transmission energy of primary user will be more compared to secondary so $C p$ is assumed to be greater than $C s$. Let $C p$ be 20 and Cs be 10. Cps and $C s p$ is taken up as 10 and 5. As the throughput of external node will be very less as it only has to report the information regarding the status of the channel it is considered as 0.5. Ep is taken up as 40 and $E s$ to be 10. The sensing energy required is taken as 0.1 and reporting energy to be 0.05 . No of channels $M$ is taken up as 3. Duration of one packet data transmission $T$ is $0.5 \mathrm{~s}$. Power of data transmission is Etx $69.5 \mathrm{~mW}$ and switching energy Esw is taken as $2 \mathrm{~mW}$ for nearby channel. In conventional cooperative sensing number of secondary users is 10 and employs Hard fusion OR rule.

Figure 1 shows graph of Normalized Delay and Throughput, wait probability $P s$ is also discussed here. The values of $P s$ are taken as 0.5 and 1 . The impact of these values is seen clearly if it decides to wait on the current channel with $P s=1$, the delay is increased with that throughput decreases suddenly. If SU decides to switch with $P s=0.5$ to idle channel the throughput increases as secondary user gets chance to transmit the data. The comparison between conventional spectrum sensing and proposed scheme is shown, for the same delay the proposed scheme achieves more throughput.

Figure 2 shows the relation between Normalized Delay and Energy consumption. SU receives the message from EN about the spectrum vacancy; it either chooses to switch or to wait. If SU decides to wait on the current channel the delay is increased with that Energy consumption remains same as it stays on current channel with its battery off for some duration $T$. If SU decides to switch to another channel then delay increases with further increase in energy consumption. Delay check can be fulfilled when the probability of waiting on the current channel without transmitting decreases, 


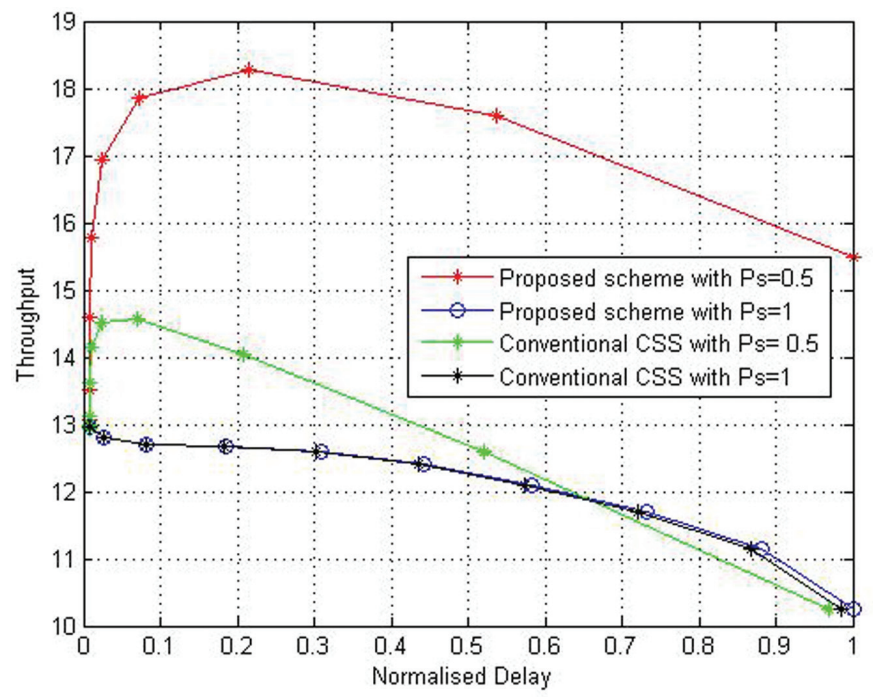

Figure 1 Normalized delay v/s throughput.

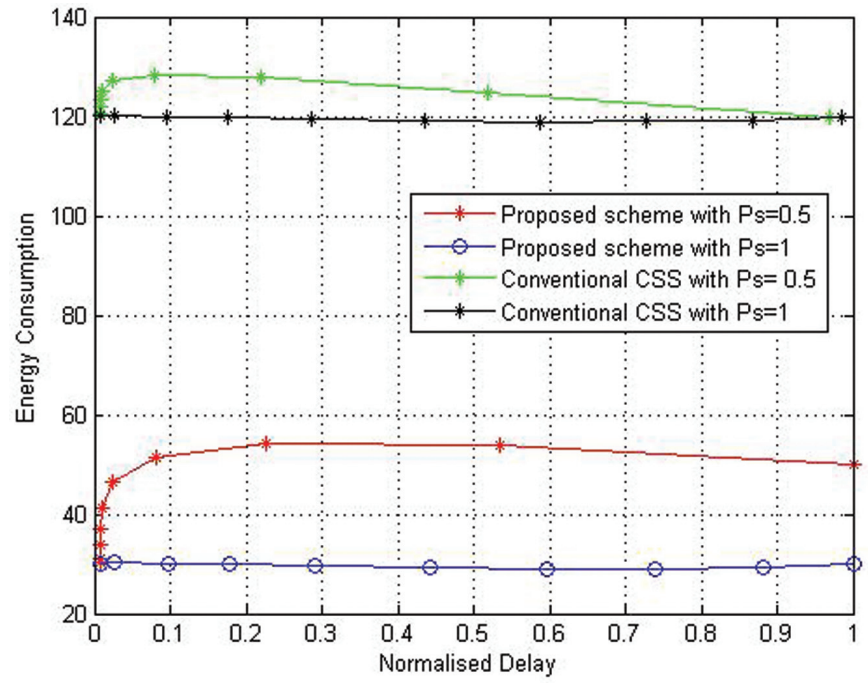

Figure 2 Normalized delay v/s energy consumption.

i.e., when a smaller $P s$ is used. However, a higher energy consumption occurs when a smaller $P s$ is used. But the difference can be seen between these two scenarios when switching takes place the energy consumed is more compared to staying on current channel. 


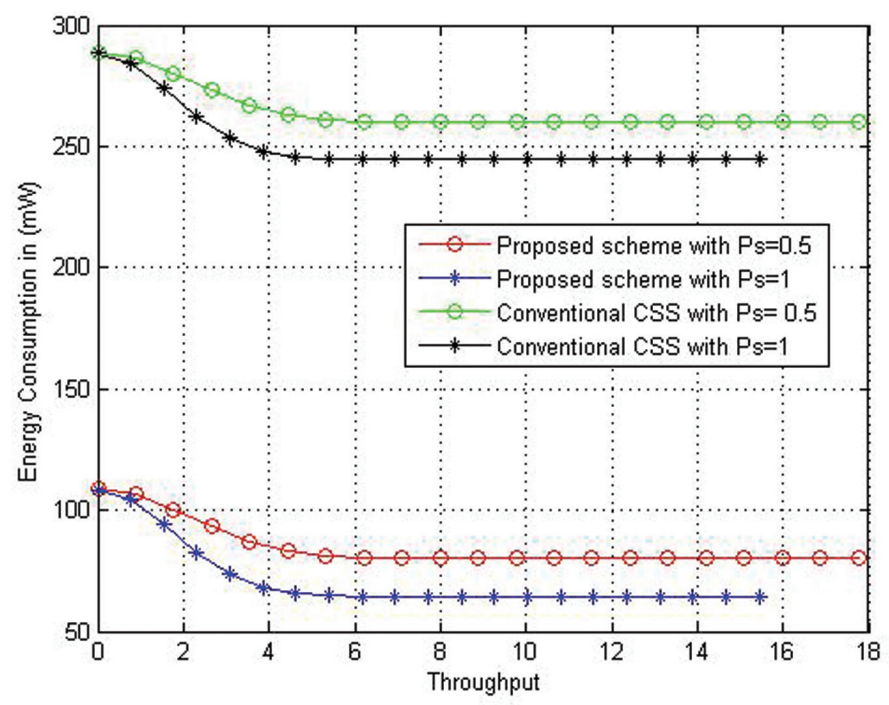

Figure 3 Throughput v/s energy consumption.

Throughput versus Energy consumption graph is shown in Figure 3. With $P s=1$, SU stays on the current channel with transmission off the energy consumed is less whereas if SU switches to idle channel with probability of $P s=0.5$ then energy consumption is more. With values of energy consumption for different $P s$ values, it is observed as throughput is increasing the energy consumption decreases. So improvement in throughput as well as in energy consumption with proposed approach is achieved compared to conventional cooperative sensing. It is also shown that the minimum energy can be achieved when the SU always chooses to wait at the current channel without transmitting.

\section{Conclusion}

A simple energy efficient multichannel cooperative sensing problem is proposed. External node does the job of spectrum sensing and reports the results to secondary users. The system model is build and the problem is formulated. $P S$ is the parameter that decides the energy consumption. The simulation results showed that the impact of $P s$ on Energy consumption, Delay and Throughput. As the external node is only involved in sensing, the energy consumed while sensing is minimized compared to conventional sensing and thus secondary users get more time for transmission so we achieve 
good tradeoff for sensing/transmission. Considering wait probability $P s=1$ minimum energy can be consumed compared to switching. Hence it satisfies the constraint of wait/switch tradeoff. Thus, with results we can say that if external sensing is employed the sensing performance is improved in terms of energy consumption and throughput.

\section{References}

[1] Commission, F. C. (2002). Spectrum policy task force report. Report ET Docket no. 02-135.

[2] Yücek, T., and Arslan, H. (2009). A survey of spectrum sensing algorithms for cognitive radio applications. IEEE Commun Surveys Tuts. 11, 116-130.

[3] Akyildiz, I. F., Lo, B. F., and Balakrishnan, R. (2011). Cooperative spectrum sensing in cognitive radio networks: a survey. Phys. Commun. J. 4, 40-62.

[4] Zhao, N., Richard Yu, F., Sun, H., and Nallanathan, A. (2013). Energyefficient cooperative spectrum sensing schemes for cognitive radio networks. EURASIP J. Wireless Commun. Netw. 1-13.

[5] Wang, S., YueWang, Coon, J. P., and Doufexi, A. (2012). Energy-efficient spectrum sensing and access for cognitive radio networks. IEEE Trans Vehic Technol. 61, 906-912.

[6] Patil, H., Patil, A. J., and Bhirud, S. G. (2015) Energy Efficient Design for External Cooperative Sensing in Cognitive Radio. in International Journal of Computer Applications 119.

[7] Peh, E., Liang, Y.-C., Guan, Y. L., and Zeng,Y. Optimization of cooperative sensing in cognitive radio networks: a sensing-throughput tradeoff view. IEEE Trans. Vehic. Technol. 58, 5294-5299.

[8] Digham, F. F., Alouini, M.-S., and Simon, M. K. (2003). "On the energy detection of unknown signals over fading channels," in Proceedings of the IEEE International Conference on Communication, Seattle, Washington, USA, pp. 3575-3579.

[9] Hua, H., Zhang, H., Yu, H., Chen, Y., and Jafarian, J. (2015). Energy-efficient design of channel sensing in cognitive radio networks. J. Comput. Electr. Eng. 42, 207-220. 


\section{Biographies}

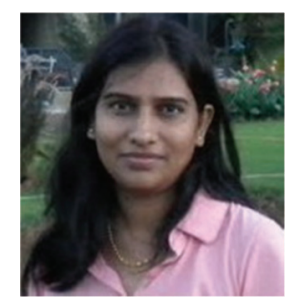

H. Patil holds a Master's Degree in Electronics and Telecommunication Engineering from the University of Mumbai, She is currently pursuing for the $\mathrm{Ph} . \mathrm{D}$. degree at University of North Maharashtra, India. Her current research interests include cognitive radio technology and wireless communications. She is life member of ISTE and IETE.

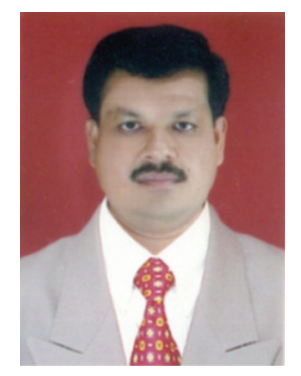

A. J. Patil was born in Jalgaon, Maharashtra, India in 1965. He received the B.E. degree in Electronics Engineering and the M.Tech. degree in Industrial Electronics and Ph.D. in Electronics Engineering from M. S. University, Baroda, Gujarat, India in 1987, 1993 and 2002 respectively. His research interests include optimal control systems, robust control/filter theory, singular systems, fuzzy control, and uncertain systems. 


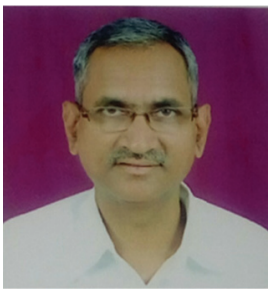

S. G. Bhirud obtained his B.E. and M.E. and Ph.D. in 1987, 1995 and 2001 respectively from S. G. G. S. College of Engineering \& Technology, Nanded, India. He worked with Bush India Ltd., and S. G. G. S. College of Engineering \& Technology, Nanded. Presently he is Professor \& Head Department of Computer Engineering \& IT at V. J. T. I. Mumbai. His areas of interest include Signal \& Image Processing, Soft Computing, Data Mining and Machine learning. He is a Life member of ISTE and CSI. 